\title{
Procedure Domain
}

National Cancer Institute

\section{Source}

National Cancer Institute. Procedure Domain. NCI Thesaurus. Code C102700.

A subject domain utilized for the submission of information encompassing and representing data, vocabulary or records related to procedure. 